\title{
Was macht die Planung? Theoretische Lockerungen mit ANT \\ Kommentar zu Lucius Burckhardts „Wer plant die Planung?“
}

Kommentare von:

Wolf Reuter, Beate Binder, Jens S. Dangschat, Monika Grubbauer, Michael Guggenheim, Stadt von Unten, Iris Dzudzek

Michael Guggenheim

Lucius Burckhardts Text „Wer plant die Planung“ ist zu Recht legendär. Schon der Titel ist ein genialer Wurf: Hinter dem Abstraktum ,Planung sind Akteure am Werk und an Akteure können Fragen gerichtet werden. Burckhardt war einer der ersten, der eine Expertenkritik formulierte und diese als ein Problem des Planungswissens, der Planungsmethoden und -theorie ausmachte und zugleich die Probleme der scheinbaren Lösung namens Partizipation ausmachte. Er betrieb Planungskritik in der Logik der Soziologie, jedoch ohne in einen banalen Soziologismus zu verfallen, der einfach böse Planer_innen guten Nutzer_innen gegenüberstellte.

40 Jahre später haben sich die von ihm diagnostizierten Planungsprobleme keineswegs in Luft aufgelöst; trotz Planung verschlechtert sich die Umwelt weiter und partizipative Formate wuchern weiter vor sich hin, ohne dass Planungskonflikte sich in Minne auflösen (Chilvers/Kearnes 2016).

Als ich gefragt wurde, ob ich einen Kommentar zu dem Text schreiben wolle, war ich sofort begeistert, weil ich meine eigene Arbeit, deren Hintergrund in der Wissenschaftsforschung und Akteur-Netzwerktheorie liegt, auch als eine Weiterschreibung Burckhardtschen Denkens sehe. Lucius Burckhardt, vermute ich, würde sich heute selbst mit Wissenschaftsforschung und Akteur-Netzwerktheorie befassen. Er wäre begeistert davon. Warum? Weil die Wissenschaftsforschung und insbesondere die Akteur-Netzwerktheorie ein Vokabular entwickelt hat, mit dem sich erstens technische Kontroversen aller Art analysieren lassen, ohne dass dafür auf festgelegte Identitäten von Berufs- und Nutzergruppen zurückgegriffen werden muss. Oder anders gesagt, weil damit das, wer in seinem Titel in ein doppeldeutiges, was ${ }^{6}$ in meinem Titel geändert werden kann, das sowohl auf die Materialien, Theorien, und Praktiken der Planung verweist als auch auf die Resultate, die daraus entstehen. Dies erlaubt es seinem organisationssoziologischen Ansatz den latenten Psychologismus auszutreiben, der das Denken von Planern hypostasiert, und ihn mit einem neuen Materialismus anzureichern. Wenn im Folgenden von ,ANT' als Kürzel für diese Form der Analyse die Rede ist, dann in einer spezifischen Form: Erstens vertrete ich, im Gegensatz zu vielen Protagonisten der ANT, die Meinung, dass es sich hierbei um eine Theorie 
handelt, das heißt eine Sprache der Weltbeschreibung, die uns eine andere Welt sehen lässt und nicht bloß um eine „Sensibilität“(Law/Singleton 2013). Zweitens fokussiere ich auf ganz wenige Elemente, die hier relevant sind, auf Kosten anderer Elemente, die historisch für die Entwicklung der ANT jedoch fundamental sind. Die ANT wurde im Kern in den 1980er Jahren von Bruno Latour, Madeleine Akrich, Michel Callon und John Law entwickelt. Alle diese Autoren interessierten sich für die Analyse von Wissenschaft als Praxis (und nicht von Architektur und Stadtplanung).[1] Zu Beginn ging es erst einmal darum nachzuvollziehen, was eigentlich Wissenschaftler und Ingenieure tun. Die wichtigste Beobachtung lautete: Sie manipulieren die Welt mit Apparaten, Technologien und Inskriptionen (oder, wie man im deutschen Sprachraum eher sagt: Medien[2]). Daraus folgte eine Absage an den Handlungsbegriff der Soziologie, und ebenso eine Absage an den Technikdeterminismus von Ingenieuren: Gesellschaft ist eine Assemblage von menschlichen und nicht-menschlichen Akteuren. Das bedeutet, dass der Handlungsbegriff tiefer gelegt wird, als dies üblicherweise in der Soziologie geschieht: Handeln wird losgelöst von Intentionen, und stattdessen an Effekten gemessen: Verkehrspolizisten, die Radarkontrollen durchführen, und Schwellen sind funktional äquivalent (um eine veraltete, aber dennoch nützliche Sprache zu verwenden): Sie machen die Autos langsamer. Aber sie kreieren je unterschiedliche Netzwerke: im ersten Fall eine Interaktion zwischen einem Polizisten, der Geschwindigkeit misst und Bußen verteilt und einem Autofahrer, im zweiten Fall zwischen einem Autofahrer, einem Auto, dessen Federung und der Betonschwelle (Latour 1992).

Könnte Lucius Burckhardt etwas von der Akteur-Netzwerktheorie lernen? Würde er den Text heute anders schreiben und anders betiteln, nachdem er sich mit einem Vokabular von Aktanten (Dingen, denen Akteurstatus zugeschrieben wird), Netzwerken (Verkettungen von menschlichen und nicht-menschlichen Akteuren) und Übersetzungen (Umwandlungen von Objekten in ein anderes Medium) bekannt gemacht hätte?[3]

Ich vermute, er würde dies zumindest in zweierlei Hinsicht tun: Erstens würde er einen prozessualeren Wissenschaftsbegriff zugrunde legen und zweitens würde er stärker auf die Rolle nicht-menschlicher Akteure im Prozess der Planung fokussieren.

Ich ignoriere hierbei die Frage, inwiefern Burckhardts organisationssoziologische und planerische Zeitdiagnose heute noch zutrifft.

Beginnen wir mit dem Wissenschaftsbegriff: Optimist, der Burckhardt war, endet er den Aufsatz mit: „Daß sich dieser Prozeß [der umfassenden Betrachtung der Planung] mit objektivem Wissen anreichert, ist zu hoffen; daß er zu einer Wissenschaft wird, ist eine Illusion."

Am Horizont schwebt die Vorstellung, Planung könne eine wirkliche Wissenschaft werden, bei der, so können wir Burckhardt interpretieren, Empirie und Theorie Hand in Hand Vorhersagen über die geplante Welt machen, die die Planung dann umsetzt. Ihr erteilt Burckhardt eine Absage, jedoch in der Hoffnung, man könne ,objektives Wissen' anreichern, um dann vielleicht die Planung zu verbessern.

Die Wissenschaftsforschung lehrt uns aber, dass Objektivität, wenn überhaupt, ein Resultat wissenschaftlicher Praktiken und nicht eine Eigenschaft von Wissen ist, die dann für irgendeine Planungstätigkeit mobilisiert werden 
kann. Überhaupt ist Burckhardts latente Gegenüberstellung von Intuition und Wissenschaft problematisch, und erst recht deren Zuordnung zu verschiedenen Professionen (Architekt_innen, Ingenieur_innen). Noch schwieriger ist die latente Annahme, die Psychologie der Planer würde bessere Lösungen verhindern, oder Planung sei ein Problem der ,Argumentation“. Die Wissenschaftsforschung hilft hier weiter, indem sie sich zuerst einmal dafür interessiert, wie denn überhaupt Planung hergestellt wird, und hierbei von normativ-kognitiven Begriffen wie Rationalität oder Objektivität absieht (bzw. deren Funktion als argumentative Ressource untersucht) und darauf fokussiert, was geschieht.

Die Akteur-Netzwerktheorie, wenn man denn so will, ist genau die Wissenschaft, die ein Wissen über die Planung hervorbringt, das planungsadäquat ist, da sie Planungswissen nicht im Kopf der Planer lokalisiert. Für Burckhardt soll es um die „umfassende Betrachtung der Art und Weise, wie Kommunen ihre Umwelt planend verändern“, gehen. Es ist zu vermuten, dass Burckhardt damit eine Art Planungswissen meint. Für die Akteur-Netzwerktheorie bedeutet dies jedoch mehr: Es meint vor allem das Arsenal an Theorien, Methoden und vor allem Medien und Techniken, die erst die Praxis ,Planung' ausmachen: Darunter sind partizipative Verfahren genauso zu zählen, wie CAD-Programme, GIS und Nachhaltigkeitsindizes.

Alle diese Objekte und Techniken sind nicht einfach Werkzeuge der Planer_innen, sondern sind Aktanten, das heißt wesentlich am Prozess beteiligt, der die Praxis Planung hervorbringt. Deswegen geht es dann gerade nicht darum den Prozess der Planung mit ,objektivem Wissen' anzureichern, sondern darum zu verstehen, wie eine spezifische Abfolge und Verkopplung von Praktiken, Techniken und Medien ,Planung' herstellt und damit auch die Selbstbeschreibungen und Resultate des Prozesses produziert und fortlaufend verändert.

Das bringt uns zum zweiten Punkt, den nicht-menschlichen Akteuren: Für Burckhardt ist das Problem der Planung ein Problem der „subjektiven Welt, " in der verschiedene Akteure leben: „Die Beplanten“wie die Planer_innen „leben in ihrer eigenen Realität“ und „wo Schwierigkeiten auftreten, handelt es sich um Übersetzungsfragen [zwischen diesen Gruppierungen]“. Damit benennt Burckhardt einen wichtigen Punkt: Planung findet nicht in einer, sondern in verschiedenen Welten statt, oder in der Terminologie der Akteur-Netzwerke: in multiplen Ontologien (Mol 2014). Ergo entsteht ein Übersetzungsproblem. Die Akteur-Netzwerktheorie würde hier zustimmen: Es handelt sich tatsächlich um Übersetzungsfragen, jedoch nicht (alleine) um solche sprachlicher Art: Die ,Realität', in der Planer_innen und Beplante leben, ist eine materialisierte Realität, und sie ist nicht einfach für Planer_ innen und Beplanten jeweils verschieden, sondern für jegliche Akteure: Alte, Junge, Fahrradfahrer_innen, Rennradfahrer_innen, Leute, denen der Friseursalon um die Ecke gehört, Leute, die gerne ein Restaurant in der Nachbarschaft eröffnen würden, die Kinderkrippe, die Macher_innen einer geolokalisierten App, den Quartierverein, die Versicherungsgesellschaft, die sich weigert ein Gebäude zu versichern, weil es einem Hochwasserrisiko ausgesetzt ist etc. Die Akteur-Netzwerktheorie würde zudem zu den verschiedenen Gruppierungen die jeweiligen Aktanten hinzufügen: Die Apps, die Friseursalone, die Strasse, die Häuser, die Feinstoffpartikel, die Autos, 
den Schattenwurf und so weiter. Die Pointe ist nun, dass sowohl die verschiedenen Akteure als auch die verschiedenen Aktanten sich je gegenseitig zur Weltkonstruktion benutzen, beziehungsweise sich je gegenseitig in verschiedene Welten übersetzen.

Die Fahrradfahrerin ist eine andere Fahrradfahrerin, wenn sie auf einem Fahrradweg fahren kann und vor dem Friseur einen Fahrradständer findet, an den sie ihr Fahrrad anschließen kann. Ja, die Nicht-Fahrradfahrerin wird zur Fahrradfahrerin, weil sie einen Fahrradweg vorfindet. Der Planer übersetzt den Autofahrer in einen Fahrradfahrer, indem er ihm einen Fahrradweg zur Verfügung stellt. Und der Freizeitfahrradfahrer wird zum Rennradfahrer, wenn der Kiesweg geteert wird. Andere Rennradfahrer_innen mieten in der Nachbarschaft Wohnungen und die 50er-Jahre-Wohnungen, die vorher als klein und schäbig galten, werden denkmalgeschützt und die Miete verdoppelt sich. Die Immobilienmakler_innen stellen Fotos der Rennradfahrer_innen in ihre Schaufenster und umranden auf ihren Stadtplänen das vorher ungenannte Straßengeviert mit einer roten Linie und geben ihm einen englisch klingenden Namen. Bei der nächsten Sitzung zur Überbauung der letzten Brachfläche bringen Aktivist_innen einen soziologischen Aufsatz mit, in dem die Rennfahrer_innen als Gentrifizierer verurteilt und als Gefahr für die Identität des Viertels bezeichnet werden.

Verschiedene Gruppen leben nicht einfach in ihrer Realität, als wäre sie ein Silo, aus dem sie nicht herauskommen, sondern die Realität ist eine, die fortlaufend neu hergestellt wird, indem andere ihnen Mittel zur Verfügung stellen, diese Realität zu verändern.

Genau deshalb kann das Übersetzungsproblem, wenn man es mit ANT versteht, nicht durch Aufklärung, consciousness raising, oder die Übersetzung in eine Laiensprache gelöst werden. Es nützt nichts, wenn sich die Planerin versucht in den Beplanten hineinzuversetzen und ihm die Logik der Planung zu erklären, genauso wenig wie umgekehrt, wenn sie versucht die Lebenswelt des Beplanten zu verstehen und aus seinen unqualifizierten, diffusen Äußerungen eine Planungslogik herauszufiltern. Vielmehr gilt es die Aufmerksamkeit auf die Übersetzungstechniken und -medien zu richten und sich zu überlegen, wie diese neue Welten kreieren, die sowohl die Planerin, als auch den Beplanten dazu bringen, eine andere Welt herzustellen.

Seit 1974, als Burckhardt den Text schrieb, lassen sich wohl zwei entgegengesetzte Bewegungen beobachten: Erstens wurden massenhaft Planungsinstrumente erfunden, deren Übersetzungsleistungen - verstanden im ANTSinne - so komplex sind, dass es immer schwieriger wird nachzuvollziehen, welche Übersetzungen diese Planungsinstrumente hervorbringen. Hierbei geht es nicht um den ,kritischen“ Einwand, die Technik operiere irgendwie im Namen einer Planungsideologie, oder um Burckhardts Vorstellung, „die Wissenschaften und Hilfswissenschaften des Stadtplaners, Stadtgeografie, Stadtsoziologie, Planungstheorie, Planungsmethodik, Planungsstrategie“ hätten zwar „Fortschritte gemacht“, aber Planung hänge dennoch „von der Person [...] ab, d[ie] plant," und laut Burckhardt handelt es sich dabei um Personen, die „eine recht eigenartige Sicht auf den Mitmenschen“ haben. Abgesehen davon, dass Burckhardts Sozialtypus des Planers (damals wahrscheinlich ein Mann) heute eher ein anderer ist: Mit ANT müssten wir eher sagen: Es geht um die simple Feststellung, dass Planung stark technisiert 
worden ist, sodass es selbst für Planer_innen kaum mehr nachvollziehbar ist, wie diese Instrumente jeweils bestimmte Planungsvarianten hervorbringen.

Als ich für meine Dissertation Umweltdienstleistungsfirmen beobachtete, begleitete ich ein Projekt, in dem versucht wurde, die Hochwasserwahrscheinlichkeit eines Flusses zu reduzieren (Guggenheim 2005). Das Projekt basierte auf einer Modellierungssoftware, mit der verschiedene Eingriffe modelliert werden konnten, wie etwa Überflutungsflächen, Bepflanzungen oder verschiedene Renaturalisierungsmaßnahmen. Nachdem die Planer_innen stundenlang für und wider diese und jene Maßnahmen gestritten und deren hypothetische Akzeptanz in der Bevölkerung diskutiert hatten, ließen sie die Software den Effekt auf die Hochwasserwahrscheinlichkeit prüfen. Dieser war dann so gering, dass sich der Aufwand aller kumulierten Maßnahmen kaum bemerkbar machte. Die Modellierungssoftware überraschte die Planer_innen, obwohl sie, ideologisch“ überzeugt davon waren, dass sie mit ihren Maßnahmen die von ihnen intendierten Ziele erreichen würden.

Zugleichwerden Planungsinstrumenteanihrem Interfacesoweitvereinfacht, dass sie zwar als Blackboxes operieren, jedoch zu Alltagsgegenständen geworden sind. The Sims und andere Weltbausimulationen, sowie eine große Zahl von Apps zur Bauplanung, zur Innenarchitektur (begonnen mit IKEA) aber auch Google Maps, haben dazu geführt, dass die Planung vor allem von Software geplant wird, die nun nicht mehr allein Planer_innen vorbehalten ist.[4] Mit dem Resultat, dass wir nun alle Planer_innen sind, ohne zu durschauen, wie wir planen und was letzten Endes durch uns geplant wird.

Diese Eigenlogik der Planungstechnologien lässt sich gerade nicht den Planer_innen oder irgendeiner Ideologie zuordnen, noch lässt sie sich als bloße Technokratie verunglimpfen. Wollte man daraus eine normative Folgerungen ziehen, dann die, dass die Planung experimentalisiert werden sollte, und zwar nicht als Frage, wer mitplant, sondern was mitplant.

\section{Endnoten}

[1] Man beachte jedoch frühe Studien zur Mobilität von Callon (1986; 1980) und dann von Latour (1996), sowie Latours Projekt zu Paris (Latour et al. 1998).

[2] Siehe zur Schnittstelle von ANT und deutschsprachiger Medientheorie (Schüttpelz/ Thielman 2013).

[3] Verschiedene Forscher in der Tradition der Wissenschaftsforschung haben sich mit dem Objekt Stadt auseinandergesetzt (Farias/Bender 2009, Gieryn 2002, 2006, Hommels 2005, Guggenheim 2008; 2016) und mittlerweile ist ANT im engeren Sinne in der Planung angekommen (Boelens 2010, Doucet 2011, Lieto/Beauregard 2015, Rydin/ Tate 2016, Kärrholm 2008).

[4] Für eine Studie, wie Laien Google Maps für ihre Zwecke nutzen, siehe Petersen (Petersen 2014).

\section{Autor_innen}

Michael Guggenheim ist Soziologe und forscht zu Umnutzungen von Gebäuden und Katastrophen.

m.guggenheim@gold.ac.uk 


\section{Literatur}

Boelens, Luuk (2010): Theorizing practice and practising theory: Outlines for an actor-relational-approach in planning. In: Planning Theory 9/1, 28-62.

Callon, Michel (1980): The State and technical innovation: A case study of the electrical vehicle in France. In: Research policy 9/4, 358-376.

Callon, Michel (1986): The sociology of an actor-network: The case of the electric vehicle. In: John Law / Arie Rip (Hg.): Mapping the Dynamics of Science and Technology. London: Macmillan, 19-34.

Chilvers, Jason / Kearnes, Matthew (2016): Remaking Participation: Science, Environment and Emergent Publics. London: Routledge.

Doucet, Isabelle (2011): Learning from Brussels. An irreductive approach to architectural and urban problématiques? In: Belgeo. Revue belge de géographie 1/2, 29-40.

Farias, Ignacio / Bender, Thomas (Hrsg.) (2009): Urban Assemblages. How Actor Network Theory Transforms Urban Studies. London: Routledge.

Gieryn, Thomas F. (2002): What buildings do. In: Theory and Society 31/1, 35-74.

Gieryn, Thomas F. (2006): City as truth-spot: Laboratories and field-sites in urban studies. In: Social Studies of Science 36/1, 5-38.

Guggenheim, Michael (2005): Organisierte Umwelt. Umweltdienstleistungsfirmen zwischen Wissenschaft, Wirtschaft und Politik. Bielefeld: Transcript.

Guggenheim, Michael (2008): Building memory. Architecture, networks and users. In: Michalis Kontopodis / Alex Kozin (Hg.): Memory Studies, Special Issue on Materializations of Times: From Memory to Imagination 2/1, 39-53.

Guggenheim, Michael (2016): Im/mutable im/mobiles. From the socio-materiality of cities towards a differential cosmopolitics. In: Anders Blok / Ignacio Farias (Hg.): Urban Cosmopolitics. Agencements, Assemblies, Atmosphere. London: Routledge, 63-81.

Hommels, Anique (2005): Studying obduracy in the city. Toward a productive fusion between technology studies and urban studies. In: Science, Technology \& Human Values 30/3, 323-351.

Kärrholm, Mattias (2008): The territorialisation of a pedestrian precinct in Malmö: Materialities in the commercialisation of public space. In: Urban Studies 45/9, 1903-1924.

Latour, Bruno (1992): Where are the missing masses. Sociology of a few mundane artefacts. In: Wiebe Bijker / John Law (Hg.): Shaping Technology - Building Society. Studies in Sociotechnical Change. Cambridge (Massachusetts): MIT Press, 225-259.

Latour, Bruno (1996): Aramis, or the Love of Technology. Cambridge (Massachusetts): Harvard University Press.

Latour, Bruno / Hermant, Emilie / Shannon, Susanna (1998): Paris ville invisible, Paris; La Découverte.

Law, John / Singleton, Vicky (2013): ANT and politics: Working in and on the world. In: Qualitative Sociology 36/4, 485-502.

Lieto, Laura / Beauregard, Robert A. (Hg.) (2015): Planning for a Material World. London: Routledge.

Mol, Annemarie (2014): A Reader's Guide to the „ontological Turn” - Part 4: Somatosphere. http://somatosphere.net/2014/o3/a-readers-guide-to-the-ontological-turn-part-4.html (letzter Zugriff am 17.02.2017).

Petersen, Katrina (2014): Producing space, tracing authority: Mapping the 2007 San Diego wildfires. In: The Sociological Review 62/S1, 91-113.

Rydin, Yvonne / Tate, Laura (2016): Actor Networks of Planning: Exploring the Influence of Actor Network Theory. London: Routledge.

Schüttpelz, Erhard / Thielman, Tristan (Hg.) (2013): Akteur-Medien-Theorie. Bielefeld: Transcript. 